\title{
Comparação da eficiência relativa de dois apetrechos de coleta de peixes em praias no baixo rio Purus, Amazonas, Brasil
}

\author{
Cleber DUARTE ${ }^{1}$, Cláudia Pereira de DEUS², Lúcia RAPP PY-DANIEL ${ }^{3}$
}

\begin{abstract}
RESUMO
Apesar das praias serem consideradas um dos sete mais importantes habitats aquáticos da Amazônia, poucos trabalhos foram realizados quanto ao levantamento de sua ictiofauna, principalmente quando utilizados diferentes apetrechos de coleta. Este trabalho teve como objetivo comparar a eficiência relativa de dois apetrechos, rede de cerco e malhadeira, ambos utilizados de forma ativa (arrasto), em três praias localizadas no baixo rio Purus. A rede de cerco apresentou maior número de espécies e indivíduos capturados, no entanto, a malhadeira apresentou maiores valores de biomassa total, representada principalmente por Siluriformes. A baixa similaridade da fauna de peixes capturada (38\%) foi devido à exclusividade de espécies coletadas com cada apetrecho, 41 com rede de cerco e 32 com malhadeira. O presente trabalho mostra a importância de se utilizar diferentes apetrechos de coleta como forma de diminuir a seletividade inerente de cada um desses aparelhos, melhorando a efetividade de amostragens em ambientes pouco estudados.
\end{abstract}

PALAVRAS-CHAVE: Ictiofauna, riqueza de espécies, malhadeira, rede de cerco.

\section{Comparison of the relative efficiency of two fishing gears in sandy beaches in lower Purus river, Amazonas, Brazil}

\begin{abstract}
Despite representing one of the seven most important aquatic habitats in the Amazon, little has been done concerning fish inventories on sandy beaches, especially related to the fishing gear used in these environments. This study aims to compare the relative efficiency of two fishing gears, seine and gill-net, used actively (trawling), on three sandy beaches on the lower reaches of Rio Purus. Seining resulted in a larger number of species and specimens, whereas for total biomass, gill-nets showed larger values, mainly represented by Siluriformes. Low similarity between samples $(38 \%)$ was due to different catches of each gear: 41 species collected only with seine and 32 only with gill-net. The present study strengthens the importance of using different fishing gears in experimental fisheries as a way to overcome the inherent selectivity of each gear, and thus improving the efficacy of sampling in poorly-known environments.
\end{abstract}

KEYWORDS: Ichthyofauna, species richness, gillnet, seine.

1 Programa de Pós-Graduação em Biologia de Água Doce e Pesca Interior, Instituto Nacional de Pesquisas da Amazônia, Caixa Postal 478, 69011-970, Manaus, AM, Brasil. (duarte@inpa.gov.br)

2 Coordenação em Biodiversidade - CBio, Instituto Nacional de Pesquisas da Amazônia, Caixa Postal 2223, 69060-001, Manaus, AM, Brasil. (claudias@inpa.gov.br)

${ }^{3}$ Coleção de Peixes, Instituto Nacional de Pesquisas da Amazônia, Caixa Postal 478, 69011-970, Manaus, AM, Brasil. (lucia.rapp@gmail.com) 
As praias dos grandes rios amazônicos são ambientes efêmeros que abrigam uma biota aquática muito diversificada, representada principalmente por espécies de peixes de pequeno porte (Ibarra e Stewart 1989; Stewart et al. 2002; Pereira et al. 2007; Duarte et al. 2010). Apesar de serem ambientes aquáticos temporários, as praias são comumente encontradas principalmente no período de águas baixas dos rios amazônicos (Santos e Ferreira 1999).

Até o momento, poucos trabalhos foram realizados quanto ao levantamento da ictiofauna de praias, sendo a rede de cerco o principal apetrecho utilizado nesses estudos (Jepsen 1997; Stewart et al. 2002; Arrington e Winemiller 2003). Em pescas artesanais em ambiente de praia, as populaçôes ribeirinhas utilizam alternativamente a malhadeira como apetrecho de pesca ativo (arrasto), devido principalmente ao seu baixo custo e a sua versatilidade (Reis e Pawson 1992). Na pesca experimental, a eficácia do uso desses dois apetrechos pode variar em relação aos parâmetros da comunidade ictíca que se pretende estudar. O presente trabalho teve como objetivo comparar a eficiência relativa de dois apetrechos, rede de cerco e malhadeira, utilizados em coletas ativas de peixes de praias, em relação à riqueza, composição de espécies, abundância $\mathrm{e}$ biomassa total.

As coletas foram realizadas em novembro de 2006, em três praias localizadas no baixo rio Purus, sendo duas na Reserva de Desenvolvimento Sustentável Piagaçu-Purus ( $04^{\circ} 27^{\prime} \mathrm{S} 61^{\circ}$ $59^{\prime} \mathrm{W}$ e $\left.04^{\circ} 30^{\prime} \mathrm{S} 62^{\circ} 03^{\prime} \mathrm{W}\right)$, e uma na Reserva Biológica do Abufari ( $05^{\circ} 22^{\prime} \mathrm{S} 63^{\circ} 04^{\prime} \mathrm{W}$ ). Foi delimitado um total de 27 pontos amostrais (nove pontos por praia), sendo cada ponto amostral correspondente a $100 \mathrm{~m}$ de praia. As coletas (arrastos) foram realizadas com rede de cerco $(5 \mathrm{~m}$ de comprimento $\mathrm{x}$ $6 \mathrm{~m}$ de altura (rede em forma de "saco"); e $5 \mathrm{~mm}$ de malha) e malhadeiras emendadas (aproximadamente $10 \mathrm{~m}$ de comprimento x $1,8 \mathrm{~m}$ de altura para cada malhadeira; malhas de tamanho 12, 40, 45, 50 e 60 mm entre nós opostos, sendo as mesmas dispostas nessa sequência de tamanhos de malha). Em cada ponto amostral foram realizados quatro arrastos não sobrepostos, sendo dois com rede de cerco e dois com malhadeira. Os quatro arrastos foram realizados manualmente e perpendiculares à linha da praia. Devido ao fato das malhadeiras emendadas apresentarem maior comprimento total $(51 \mathrm{~m})$, os arrastos foram realizados com auxilio de uma canoa à remo, de forma que uma das extremidades era levada da margem até uma distância de aproximadamente 10 metros e posteriormente arrastada de volta a margem (formando-se um "arco"). Os arrastos com rede de cerco foram realizados até uma profundidade de aproximadamente $1,5 \mathrm{~m}$. Os exemplares coletados foram eutanasiados com eugenol e posteriormente fixados em solução de formalina $10 \%$. Após triagem, identificação e contagem das espécies e exemplares de peixes, o material foi colocado em álcool $70 \%$ e registrado na Coleçâo de Peixes do INPA. Análises de similaridade foram realizadas utilizando-se o índice qualitativo de Jaccard. A partir dos dados de riqueza, abundância e biomassa total obtidos por ponto de coleta (27), foi aplicado inicialmente um teste de homocedasticidade (teste de Levene) para verificar se os mesmos atendiam às premissas para a utilizaçáo de uma análise paramétrica. $\mathrm{O}$ teste de homocedasticidade não foi atendido, sendo realizada, portanto, uma análise não-paramétrica de Mann-Whitney (teste- $\mathrm{U})$ para se testar as diferenças $(\mathrm{p}<0,05)$ entre malhadeira e rede de cerco. Para as espécies coletadas com ambos os apetrechos, os valores de abundância e biomassa foram comparados pareadamente, utilizando análise nãoparamétrica de Wilcoxon (teste- W). Para essas análises foi utilizado o programa estatístico PAST 2.03.

Nas três praias coletadas foram registradas 118 espécies, com um total de 9.486 peixes. A ordem mais rica foi Characiformes com 51 espécies (43\%), seguida por Siluriformes com 41 (35\%). A família mais rica foi Characidae, com 28 espécies, seguida por Pimelodidae com 13. A coleta com rede de cerco resultou em uma maior captura total de exemplares (8.598 vs. 888) e um maior número de espécies registradas (86 vs. 77). Em relação à biomassa total, malhadeira apresentou maiores valores $(212.070 \mathrm{~g}$ vs. $39.452 \mathrm{~g})$, representado principalmente por Siluriformes de maior porte ( $>10 \mathrm{~cm} \mathrm{CP}$ ) (Figura 1).

Foi observada diferença significativa na abundância $(\mathrm{U}=$ 68,5; $\mathrm{p}<0,0001 ; \mathrm{n}=27)$, riqueza $(\mathrm{U}=76 ; \mathrm{p}<0,0001 ; \mathrm{n}=$ 27) e biomassa $(U=123 ; \mathrm{p}<0,0001 ; \mathrm{n}=27)$ capturada com cada apetrecho (Figura 2). Na análise pareada, no entanto, utilizando-se as espécies coletadas com ambos os apetrechos (45) (Tabela 1), o teste Wilcoxon (teste- W) revelou diferença significativa para a biomassa $(W=710 ; p=0,0298 ; n=45)$, mas não para os valores de abundância $(\mathrm{W}=510 ; \mathrm{p}=0,3009$; $\mathrm{n}=45$ ). Entretanto, foi observado que algumas espécies são bem mais abundantes na rede de cerco (Triportheus cf. albus,

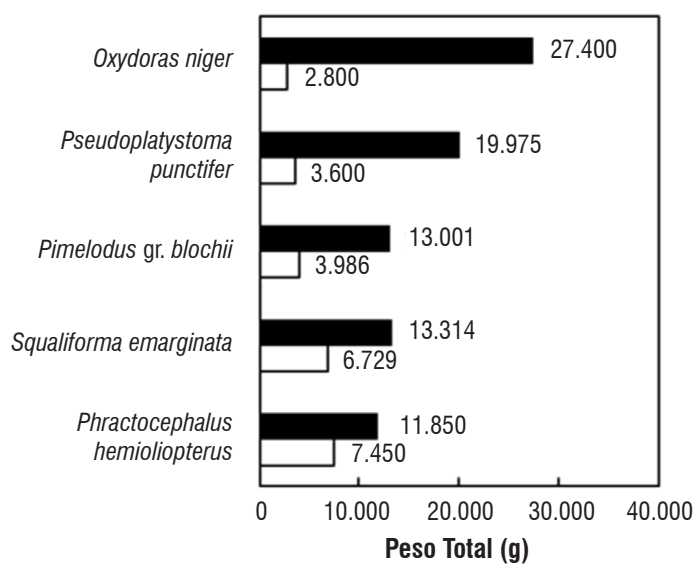

Figura 1 - Ordenamento das espécies com os maiores valores de biomassa total (g) coletadas com malhadeira (barra preta) e rede de cerco (barra branca). 
Tabela 1 - Lista de espécies e número de exemplares coletados nas três praias do baixo rio Purus com os apetrechos rede de cerco (R) e malhadeira (M).

\begin{tabular}{|c|c|c|c|c|c|c|c|c|c|c|c|}
\hline Espécie & $\mathrm{R}$ & M & Espécie & $\mathrm{R}$ & $\mathrm{M}$ & Espécie & $\mathrm{R}$ & M & Espécie & $\mathrm{R}$ & M \\
\hline $\begin{array}{l}\text { Acestrorhynchus } \\
\text { falcatus }\end{array}$ & 3 & 1 & Eigenmannia limbata & 16 & 0 & Moenkhausia cf. megalops & 6 & 0 & Pygocentrus nattereri & 0 & 9 \\
\hline $\begin{array}{l}\text { Acestrorhynchus } \\
\text { falcirostris }\end{array}$ & 0 & 2 & Eigenmannia macrops & 636 & 0 & Moenkhausia cf. oligolepis & 1 & 0 & Rhabdolichops sp.1 & 26 & 0 \\
\hline $\begin{array}{l}\text { Acestrorhynchus } \\
\text { microlepis }\end{array}$ & 0 & 2 & Geophagus cf. proximus & 955 & 2 & Moenkhausia gr. lepidura & 1339 & 0 & Rhabdolichops sp.2 & 1 & 0 \\
\hline Ageneiosus atronasus & 6 & 9 & $\begin{array}{c}\text { Gymnorhamphichthys cf. } \\
\text { rondoni }\end{array}$ & 7 & 0 & Mylossoma aureum & 0 & 161 & Rhaphiodon vulpinus & 0 & 7 \\
\hline Ageneiosus inermis & 1 & 5 & Hemidoras morrisi & 0 & 2 & Mylossoma duriventre & 0 & 105 & $\begin{array}{c}\text { Rhytiodus } \\
\text { argenteofuscus }\end{array}$ & 0 & 1 \\
\hline Ageneiosus ucayalensis & 0 & 5 & Hemidoras stenopeltis & 16 & 17 & Ochmacanthus reinhardtii & 11 & 0 & Rhytiodus microlepis & 1 & 0 \\
\hline Agoniates anchovia & 4 & 2 & $\begin{array}{l}\text { Hemiodontichthys } \\
\text { acipenserinus }\end{array}$ & 6 & 4 & Osteoglossum bicirrhosum & 0 & 41 & $\begin{array}{l}\text { Rineloricaria } \\
\text { phoxocephala }\end{array}$ & 2 & 0 \\
\hline Anchoviella cf. carrikeri & 101 & 0 & $\begin{array}{l}\text { Hemiodus "microlepis- } \\
\text { Iongo" }\end{array}$ & 0 & 3 & Oxydoras niger & 2 & 25 & Rivulus ornatus & 1 & 0 \\
\hline Aphyocharax alburnus & 111 & 0 & Hemiodus argenteus & 3 & 9 & Pachyurus cf. gabrielensis & 27 & 0 & Roeboides affinis & 12 & 2 \\
\hline Astyanax sp. & 1 & 0 & Hemiodus immaculatus & 0 & 1 & Paravandellia sp. & 49 & 3 & Roeboides myersi & 5 & 3 \\
\hline $\begin{array}{c}\text { Auchenipterus } \\
\text { brachyurus }\end{array}$ & 0 & 1 & $\begin{array}{l}\text { Hemisorubim } \\
\text { platyrhynchos }\end{array}$ & 4 & 3 & Pellona castelnaeana & 0 & 2 & Schizodon fasciatus & 6 & 5 \\
\hline Biotodoma sp. & 1 & 0 & Henonemus punctatus & 9 & 0 & Pellona flavipinnis & 0 & 3 & $\begin{array}{l}\text { Semaprochilodus } \\
\text { insignis }\end{array}$ & 1 & 1 \\
\hline Boulengerella cuvieri & 3 & 1 & Hoplias malabaricus & 12 & 2 & $\begin{array}{c}\text { Phractocephalus } \\
\text { hemioliopterus }\end{array}$ & 7 & 9 & $\begin{array}{l}\text { Semaprochilodus } \\
\text { taeniurus }\end{array}$ & 3 & 5 \\
\hline Boulengerella maculata & 2 & 0 & $\begin{array}{l}\text { Hydrolycus } \\
\text { scomberoides }\end{array}$ & 0 & 2 & Piaractus brachypomus & 0 & 4 & Serrasalmus rhombeus & 0 & 3 \\
\hline $\begin{array}{l}\text { Calophysus } \\
\text { macropterus }\end{array}$ & 1 & 3 & Hypoclinemus mentalis & 23 & 0 & Pimelodella steindachneri & 55 & 3 & $\begin{array}{l}\text { Serrasalmus } \\
\text { spilopleura }\end{array}$ & 0 & 1 \\
\hline Centromochlus heckelli & 0 & 23 & $\begin{array}{l}\text { Hypophthalmus } \\
\text { edentatus }\end{array}$ & 0 & 1 & Pimelodus gr. blochii & 293 & 181 & Sorubim elongatus & 0 & 4 \\
\hline Centromochlus sp. & 4 & 0 & Hypoptoma incognitum & 2 & 5 & Pinirampus pirinampu & 0 & 4 & $\begin{array}{l}\text { Sorubimichthys } \\
\text { planiceps }\end{array}$ & 1 & 3 \\
\hline Cetopsis sp. & 1 & 1 & Ilisha amazonica & 312 & 0 & $\begin{array}{l}\text { Plagioscion cf. } \\
\text { squamosissimus }\end{array}$ & 968 & 1 & $\begin{array}{l}\text { Squaliforma } \\
\text { emarginata }\end{array}$ & 47 & 37 \\
\hline Chalceus erythrurus & 29 & 2 & Leiarius marmoratus & 0 & 2 & Platynematichthys notatus & 0 & 2 & $\begin{array}{l}\text { Steindachnerina } \\
\text { bimaculata }\end{array}$ & 3 & 3 \\
\hline Charax sp. & 2 & 0 & Leptagoniates pi & 6 & 0 & Plectrochilus diabolicus & 1 & 0 & Sternachella sp. & 2 & 0 \\
\hline Cheirocerus goeldii & 116 & 0 & Loricaria cataphracta & 2 & 2 & Potamorhina latior & 4 & 27 & Sturisoma guentheri & 4 & 2 \\
\hline Cichla monoculus & 0 & 2 & $\begin{array}{l}\text { Loricariichthys } \\
\text { nudirostris }\end{array}$ & 2 & 4 & Prionobrama filigera & 73 & 0 & $\begin{array}{l}\text { Tetragonopterus } \\
\quad \text { argenteus }\end{array}$ & 1 & 0 \\
\hline Colomesus asellus & 404 & 1 & Lycengraulis batesii & 483 & 2 & Pristigaster cayana & 0 & 1 & Thoracocharax securis & 0 & 2 \\
\hline Crenicichla gr. reticulata & 1 & 0 & $\begin{array}{l}\text { Microphilypnus } \\
\text { amazonicus }\end{array}$ & 1 & 0 & Pristigaster whiteheadi & 439 & 0 & Trachydoras nattereri & 69 & 8 \\
\hline Crenicichla sp. & 3 & 0 & $\begin{array}{c}\text { Microschemobrycon } \\
\text { geisleri }\end{array}$ & 328 & 0 & Prochilodus nigricans & 0 & 24 & $\begin{array}{l}\text { Trachydoras } \\
\text { steindachneri }\end{array}$ & 0 & 9 \\
\hline $\begin{array}{l}\text { Ctenobrycon } \\
\text { hauxwellianus }\end{array}$ & 28 & 1 & Microschemobrycon sp. & 1 & 0 & Pseudoloricaria cf. punctata & 107 & 0 & Triportheus angulatus & 2 & 22 \\
\hline Curimatella alburna & 0 & 1 & $\begin{array}{l}\text { Moenkhausia "aff. } \\
\text { megalops" }\end{array}$ & 1 & 0 & Pseudoplatystoma punctifer & 2 & 18 & Triportheus auritus & 0 & 3 \\
\hline Curimatella dorsalis & 2 & 0 & Moenkhausia cf. browni & 12 & 0 & Pseudoplatystoma tigrinum & 1 & 4 & Triportheus cf. albus & 1161 & 1 \\
\hline \multirow[t]{2}{*}{ Cynodon gibbus } & 3 & 1 & Moenkhausia cf. jamesi & 26 & 9 & Pseudotylosurus sp. & 5 & 0 & Vandellia cirrhosa & 161 & 3 \\
\hline & & & & & & Pterygoplichthys pardalis & 1 & 3 & Vandellia sanguinea & 8 & 0 \\
\hline
\end{tabular}



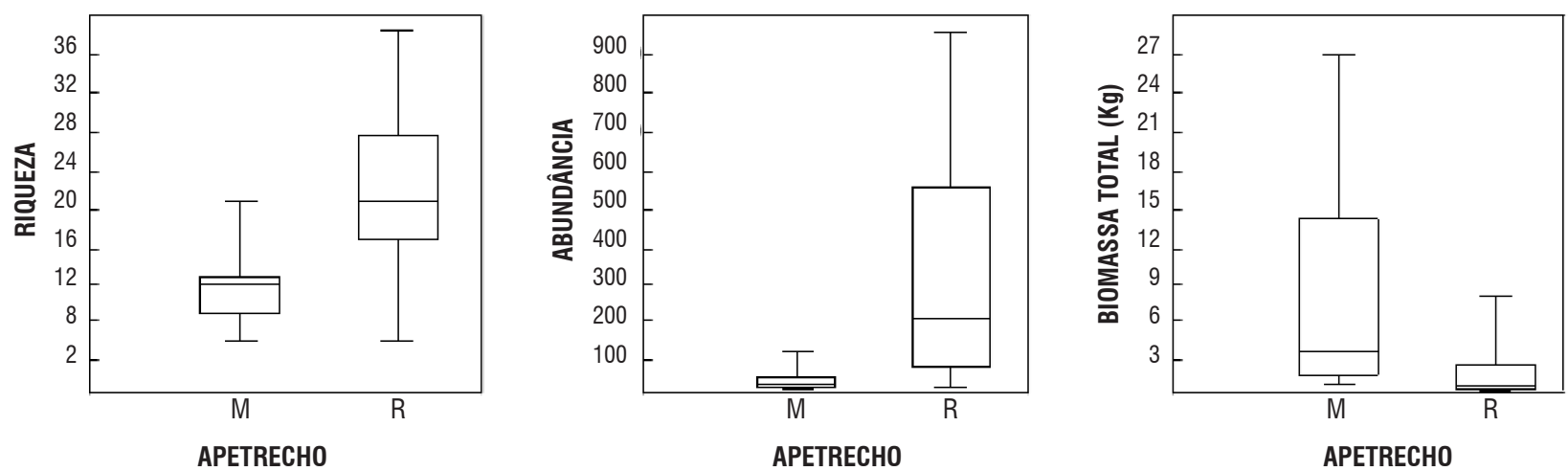

Figura 2 - Valores de riqueza de espécies, abundância e biomassa obtidos com os apetrechos malhadeira (M) e rede de cerco (R), representados com a médiana (linha horizontal dentro da caixa) e máximo/mínimo (linhas curtas horizontais fora da caixa).

Geophagus cf. proximus e Plagioscion cf. squamosissimus). Apesar de contrastante, o resultado do teste Wilcoxon (teste- W) foi devido à maior parte das espécies coletadas em comum terem apresentado uma abundância semelhante com ambos os apetrechos (Schizodon fasciatus, Semaprochilodus insignis, S. taeniurus, Steindachnerina bimaculata) (Tabela 1).

A análise de Similaridade revelou pouca semelhança (38\%) na composiçáo de espécies de peixes entre os apetrechos. Esse resultado se deve principalmente ao número de espécies coletadas exclusivamente com cada apetrecho, ou seja, 41 espécies (35\%) com rede de cerco e 32 (27\%) com malhadeira. Nas coletas com rede de cerco foi observada uma maior abundância de espécies de pequeno porte (Moenkhausia gr. lepidura) e indivíduos juvenis (Triportheus cf. albus, Geophagus cf. proximus e Plagioscion cf. squamosissimus). Esse resultado corrobora estudos anteriores, que verificaram que esse ambiente é ocupado principalmente por espécies de pequeno porte (Ibarra e Stewart 1989; Stewart et al. 2002; Pereira et al. 2007; Duarte et al. 2010). Para a maioria dessas espécies, as praias podem servir como local de proteção el ou alimentação (Goulding 1997; Lowe-McConnell 1999). Entretanto, nas coletas com malhadeiras foi observada uma abundância de espécies de maior porte, como o bagre Platynematichthys notatus e o tucunaré Cichla monoculus (>10 $\mathrm{cm}$ CP); além de espécies de interesse comercial, como os pacus Mylossoma aureum e $M$. duriventre, que também apresentaram uma alta abundância (Tabela 1). Entre as possíveis causas para essa diferença observada entre os apetrechos estão: (1) a maior seletividade das malhadeiras, possibilitando a captura de espécies maiores; (2) o pequeno tamanho da rede de cerco e consequentemente uma menor área de alcance, possibilitando a captura de espécies menores e com menor capacidade de fuga; (3) diferença entre os tamanhos de malhas utilizadas nos dois apetrechos e (4) diferença entre as amplitudes de coluna d'água amostradas (maior área de coluna d'água com malhadeira).
Apesar dos poucos trabalhos realizados utilizando diferentes apetrechos de coleta de peixes para o levantamento da ictiofuna de praias de rios (Barthem e Goulding 1997; Arrington e Winemiller 2003), arrastos com malhadeira são utilizados em praias para a captura de bagres do gênero Pseudoplatystoma (Barthem e Goulding 1997). Já Arrington e Winemiller (2003), durante as coletas de praias no rio Cinaruco, Venezuela, utilizaram tarrafa para capturar espécies, como o tucunaré Cichla spp., que evitam a rede de cerco durante o dia. Comparaçóes de métodos de pesca realizadas em diversos tipos de ambientes aquáticos têm demonstrado que os melhores resultados, em termos de levantamento da ictiofauna e de estimativas de abundância, são obtidos com uso de métodos pouco seletivos, como a pesca elétrica (Pugh e Schramm 1998), porém de custo mais elevado. Por outro lado, a comparação de métodos mais simples (e necessariamente mais seletivos) geralmente resulta na conclusão de que tais métodos produzem resultados complementares, e deveriam ser utilizados em conjunto, mesmo em ambientes mais restritos como riachos (Goldstein 1978; Ribeiro e Zuanon 2006). Apesar das diferenças observadas entre os apetrechos, com uma maior riqueza e abundância observada para a rede de cerco, os resultados obtidos no presente estudo também apontam para a necessidade de utilização de diferentes apetrechos de coleta para garantir a efetividade das amostragens.

O presente resultado pode ter apresentado limitaçóes quanto ao número de réplicas em um único período hidrológico (seca), porém, os diferentes pontos amostrados apresentaram padróes semelhantes. Estudos futuros, portanto, são necessários para um melhor entendimento da dinâmica biológica desse ambiente.

\section{AGRADECIMENTOS}

Ao Programa de Pós-Graduação em Biologia de Água Doce e Pesca Interior (BADPI/INPA); ao CNPq pela concessão da bolsa; ao IBAMA pela licença de coleta (n.027/2007), ao 
Instituto Piagaçu pelo apoio logístico e aos companheiros de coleta José Rabello Neto, Rodrigo Neves dos Santos, Sidinéia Amadio, Odirlene Ribeiro e Wellington Pedroza. A Cristhiana Röpke, pela ajuda nas análises estatísticas.

\section{BIBLIOGRAFIA CITADA}

Arrington, D. A.; Winemiller, K. O. 2003. Diel changeover in sand-beach fish assemblages in a Neotropical floodplain river. Environmental Biology of Fishes, 63: 442-459.

Barthem, R. B.; Goulding, M. 1997. The catfish connection: ecology, migration and conservation of Amazon predators. Columbia University Press, New York, USA. 144 p.

Duarte, C.; Rapp Py-Daniel, L. H.; Deus, C. P. 2010. Fish assemblages in two sandy beaches in lower Purus River, Amazonas, Brazil. Iheringia, Série Zoologia, 100: 319-328.

Goldstein, R. M. 1978. Quantitative comparison of seining and underwater observation for stream fishery surveys. The Progressive Fish-Culturist, 40: 108-111.

Goulding, M. 1997. História Natural dos Rios Amazônicos. Sociedade Civil Mamirauá/CNPq/Rainforest Alliance. Brasília. 208 pp.

Ibarra, M.; Stewart. J. D. 1989. Longitudinal zonation of sandy beach fishes in the Napo river basin, eastern Ecuador. Copeia, 2: 364-381.

Jepsen, D. B. 1997. Fish species diversity in sand bank habitats of neotropical river. Environmental Biology of Fishes, 49: 449-460.
Lowe- McConnell, R.H. 1999. Ecological studies of tropical fish communities. EDUSP, São Paulo, SP. 534 pp.

Pereira, P. R.; Agostinho, C. S.; Oliveira, R. J.; Marques, E. E. 2007. Trophic guilds of fishes in sandbank habitats of a Neotropical river. Neotropical Ichthyology, 5(3):399-404.

Pugh, L. L.; Schramm, H. L. 1998. Comparation of electrofishing and hoopnetting in lotic habitats of the Lower Mississipi River. North American Journal of Fisheries Management, 18: 649-656.

Reis, E.G.; Pawson, M.G. 1992. Determination of gillnet selectivity for bass (Dicentrarchus labrax L.) using commercial catch data. Fisheries Research, 13: 173-187.

Ribeiro, O. M.; Zuanon, J. 2006. Comparação da eficiência de dois métodos de coleta de peixes em igarapés de terra firme da Amazônia Central. Acta Amazonica, 36: 389 -394.

Santos, G.M.; Ferreira, E. 1999. Amazonian Fish, In: LoweMcConnell, R. H (Ed). Ecological studies of tropical fish communities. EDUSP, São Paulo, SP, p. 220-250.

Stewart, J. D.; Ibarra, M.; Barriga-Salazar, R. 2002. Comparison of deep-river and adjacent sandy-beach fish assemblages in the Napo river basin, eastern Ecuador. Copeia 2: 333-343.

Recebido em 01/03/2012

Aceito em 07/02/2013 
